\title{
Editorial
}

\section{Laboratory Medicine 2016}

\author{
Mina Hur, ${ }^{1}$ Patrizia Cardelli, ${ }^{2}$ and Giulio Mengozzi $^{3}$ \\ ${ }^{1}$ Department of Laboratory Medicine, Konkuk University School of Medicine, 120-1 Neungdong-ro, Hwayang-dong, Gwangjin-gu, \\ Seoul 05030, Republic of Korea \\ ${ }^{2}$ Clinical and Molecular Medicine Department Azienda Ospedaliera Sant'Andrea, School of Medicine and Psychology, \\ Sapienza University, Rome, Italy \\ ${ }^{3}$ Laboratory Medicine Department, Clinical Biochemistry Laboratory, “Città della Salute e della Scienza” University Hospital of Turin, \\ Turin, Italy
}

Correspondence should be addressed to Mina Hur; dearmina@hanmail.net

Received 5 December 2016; Accepted 6 December 2016

Copyright (C) 2016 Mina Hur et al. This is an open access article distributed under the Creative Commons Attribution License, which permits unrestricted use, distribution, and reproduction in any medium, provided the original work is properly cited.

Laboratory medicine is the bridging filed between basic research and clinical practice. Numerous candidate research findings and assays should be filtered, modified, and validated in terms of their analytical and clinical utility before being considered clinically useful. Clinical laboratory tests have essential roles in clinical decision-making from diagnosis to monitoring; accordingly, laboratory medicine is related to every specialty in clinical filed.

This is the third special issue on laboratory medicine, succeeding the issues of Laboratory Medicine and Laboratory Medicine 2014. This issue includes seven research papers covering a wide variety of interesting laboratory-related topics.

Three papers deal with the growing area of molecular diagnostic applications. The paper by Y. Chung et al. demonstrated the usefulness of multiplex real-time PCR for simultaneous pathogen detection and resistance profiling of Staphylococcal bacteremia. This direct multiplex realtime PCR assay of positive blood cultures containing Grampositive cocci in clusters could provide essential information at the critical point of infection with a turnaround time of no more than $4 \mathrm{~h}$. The paper by T. S. Kim et al. explored the significance of "not detected but amplified" (NDBA) results by real-time PCR method for human papilloma virus (HPV) DNA detection. They concluded that NDBA results in realtime PCR should be regarded as equivocal, not negative, and also addressed the adjustment of cut-off Ct value for HPV types for the appropriate result interpretation. Q.-Q. Zheng et al. performed a clinical and molecular study and identified a large SLC25A13 deletion via sophisticated molecular analyses using peripheral blood lymphocytes in an infant with neonatal intrahepatic cholestasis caused by citrin deficiency.

The other three papers came from the conventional areas of hematology, immunoassay, and microbiology. M. Y. Lee et al. evaluated mean platelet volume (MPV) in tuberculosis (TB) patients and showed that MPV can be an inflammatory marker to determine the disease activity in TB patients. The paper by J. Samardzic et al. demonstrated that different vancomycin immunoassays contribute to the variability in vancomycin trough measurements in neonates. They suggested the impact of vancomycin immunoassays in neonatal matrix, supporting a switch to more advanced techniques (LC MS/MS). The other paper by S. H. Kim et al. explored the serotype distribution and antimicrobial resistance of Streptococcus pneumoniae isolates causing invasive and noninvasive pneumococcal diseases in Korea for seven years.

The last paper by J. S. Heo et al. covers the basic field of laboratory medicine. They demonstrated that poly-L-lysine provides favorable microenvironment for mesenchymal stem cells (MSCs) culture by reversing the replicative senescence that is typical of MSCs cultured in vitro. This method will 
significantly contribute to effective preparation of MSCs for cellular therapy.

We hope that this special issue would solidify the unique value of this journal and contribute to the scientific development in this field.

Mina Hur

Patrizia Cardelli

Giulio Mengozzi 

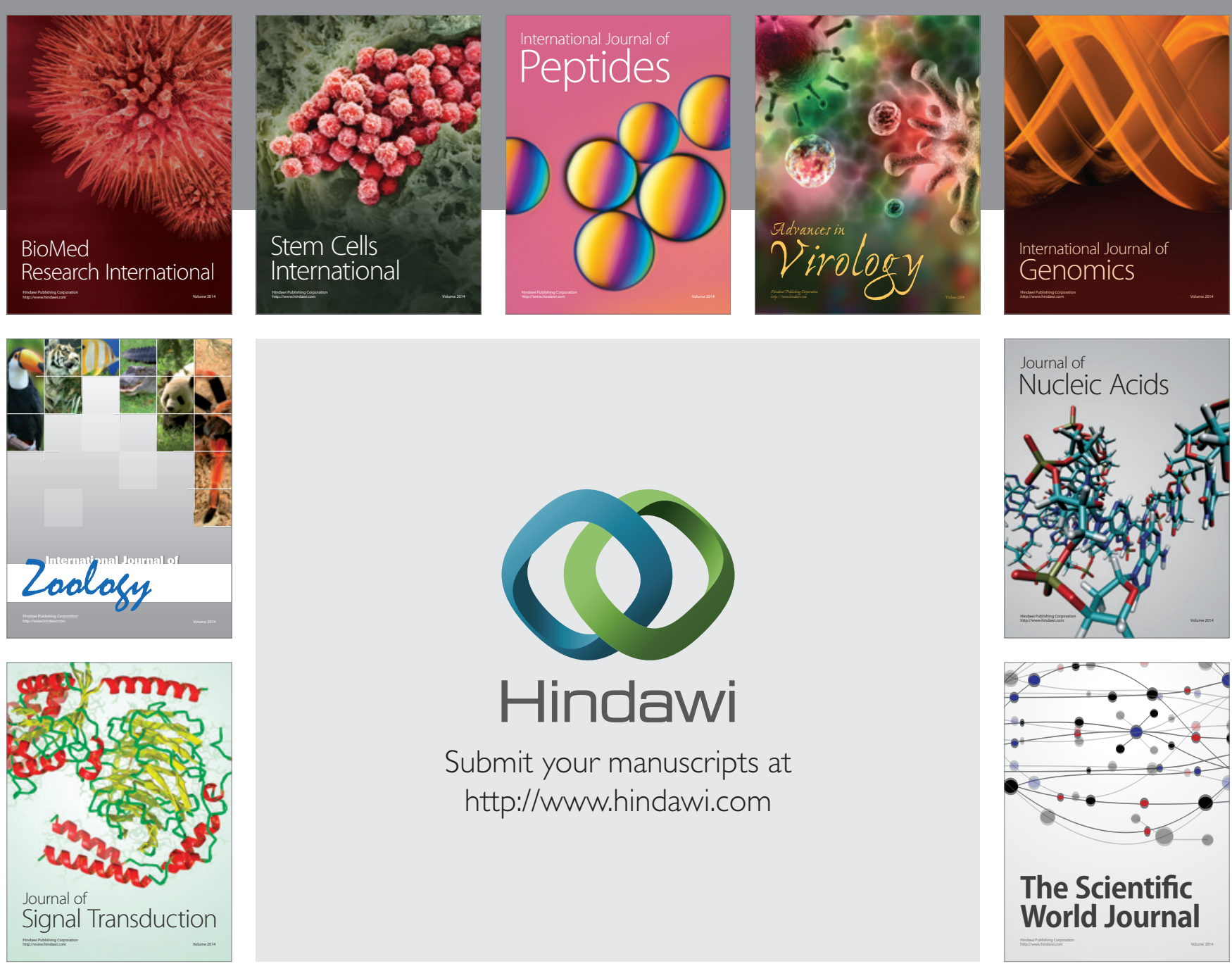

Submit your manuscripts at

http://www.hindawi.com
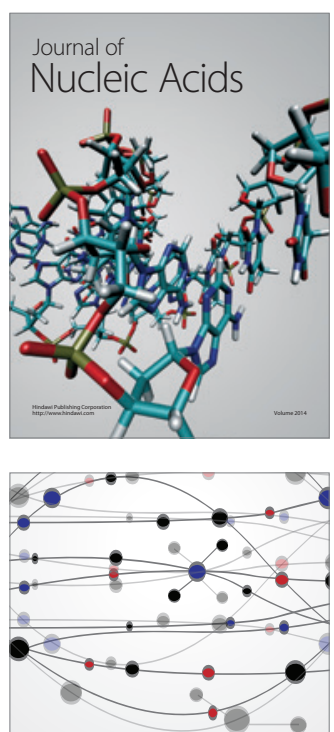

The Scientific World Journal
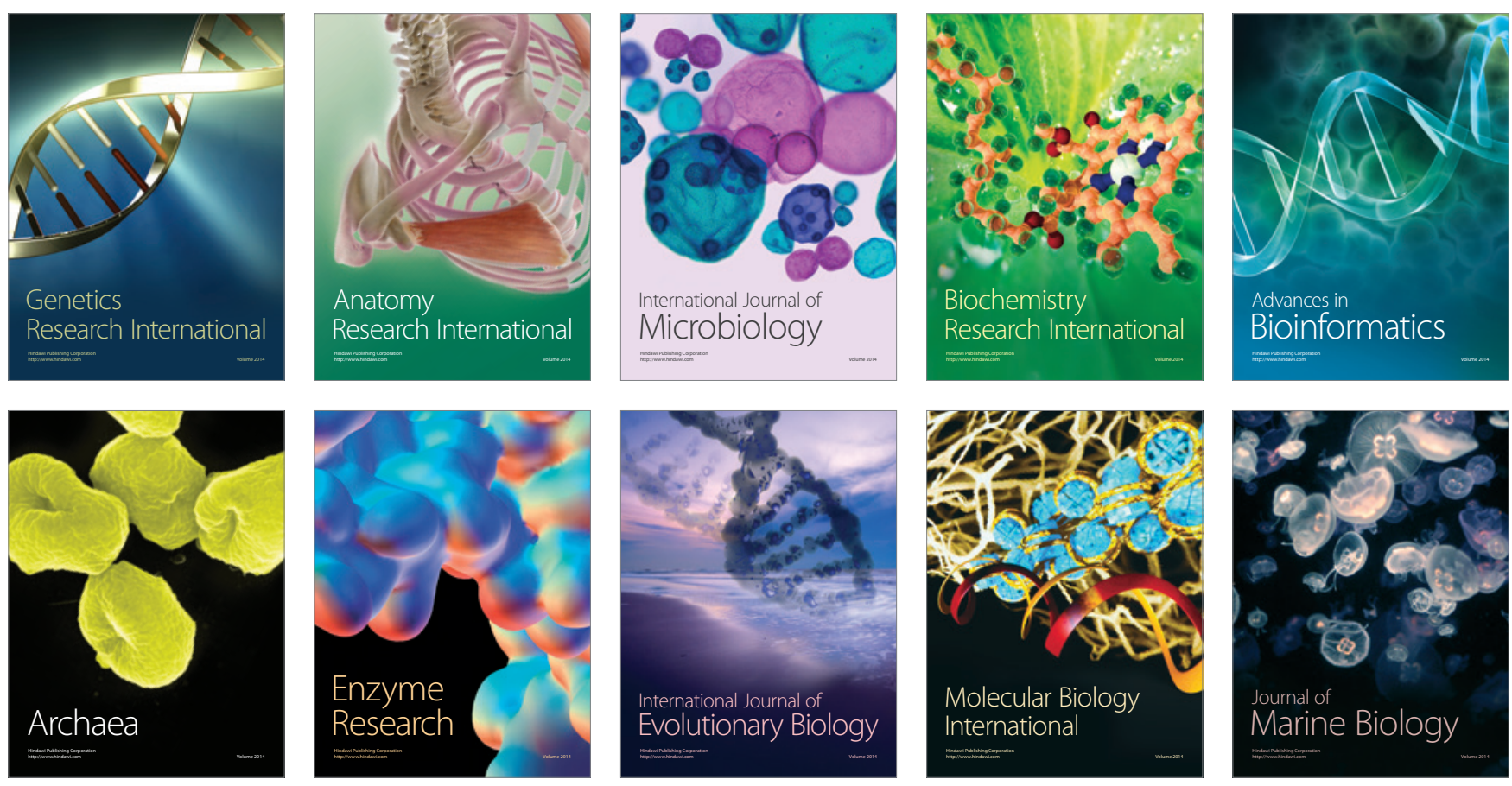